OPEN ACCESS

Edited by:

Corette J. Wierenga,

Utrecht University, Netherlands

Reviewed by:

Chiayu Chiu,

University of Valparaíso, Chile

Harold MacGillavry,

Utrecht University, Netherlands

*Correspondence:

Beulah Leitch

beulah.leitch@otago.ac.nz

Received: 09 October 2017 Accepted: 15 December 2017

Published: 22 December 2017

Citation:

Adotevi NK and Leitch B (2017)

Synaptic Changes in AMPA Receptor

Subunit Expression in Cortical

Parvalbumin Interneurons

in the Stargazer Model of Absence

Epilepsy.

Front. Mol. Neurosci. 10:434.

doi: 10.3389/fnmol.2017.00434

\section{Synaptic Changes in AMPA Receptor Subunit Expression in Cortical Parvalbumin Interneurons in the Stargazer Model of Absence Epilepsy}

\author{
Nadia K. Adotevi and Beulah Leitch* \\ Department of Anatomy, Brain Health Research Centre, School of Biomedical Sciences, University of Otago, Dunedin, \\ New Zealand
}

Feedforward inhibition is essential to prevent run away excitation within the brain. Recent evidence suggests that a loss of feed-forward inhibition in the corticothalamocortical circuitry may underlie some absence seizures. However, it is unclear if this aberration is specifically linked to loss of synaptic excitation onto local fastspiking parvalbumin-containing $\left(\mathrm{PV}^{+}\right)$inhibitory interneurons, which are responsible for mediating feedforward inhibition within cortical networks. We recently reported a global tissue loss of AMPA receptors (AMPARs), and a specific mistrafficking of these AMPARs in $\mathrm{PV}^{+}$interneurons in the stargazer somatosensory cortex. The current study was aimed at investigating if cellular changes in AMPAR expression were translated into deficits in receptors at specific synapses in the feedforward inhibitory microcircuit. Using western blot immunolabeling on biochemically isolated synaptic fractions, we demonstrate a loss of AMPAR GluA1-4 subunits in the somatosensory cortex of stargazers compared to non-epileptic control mice. Furthermore, using double post-embedding immunogold-cytochemistry, we show a loss of GluA1-4-AMPARs at excitatory synapses onto cortical $\mathrm{PV}^{+}$interneurons. Altogether, these data indicate a loss of synaptic AMPAR-mediated excitation of cortical $\mathrm{PV}^{+}$inhibitory neurons. As the cortex is considered the site of initiation of spike wave discharges (SWDs) within the corticothalamocortical circuitry, loss of AMPARs at cortical $\mathrm{PV}^{+}$interneurons likely impairs feed-forward inhibitory output, and contributes to the generation of SWDs and absence seizures in stargazers.

Keywords: absence epilepsy, stargazin, AMPA receptor, parvalbumin, feed-forward inhibition, somatosensory cortex

\section{INTRODUCTION}

Absence epilepsy is the most common early-onset epilepsy, accounting for about $10-17 \%$ of all pediatric epilepsies (Matricardi et al., 2014). It is a non-convulsive, generalized genetic epilepsy characterized by sudden, brief loss of consciousness, which can occur 100s of times a day (Berg et al., 2010). Due to the disruptive nature of the seizures, brought on by the many periods of abrupt 'absence', some affected children exhibit academic difficulties, psychosocial problems, as well as an increased risk of injury during physical activities (Wirrel et al., 1996; Caplan et al., 2008). 
In order to reduce these poor outcomes, pharmacological treatment with anti-seizure medication is recommended to reduce the frequency of seizures (Matricardi et al., 2014). Despite years of extensive research into seizure mechanisms, this has not translated into the development of novel anti-epileptic drugs (AEDs) with increased efficacy for treatment (Loscher, 2017). As such, ethosuximide, which was introduced almost 60 years ago (Zimmerman and Burgemeister, 1958), specifically for the treatment of absence seizures (Goren and Onat, 2007), is still the optimal initial monotherapy recommended for treatment of childhood absence epilepsy. However, according to the most recent randomized control trial conducted in accordance with the treatment guidelines of the International League Against Epilepsy (ILAE), ethosuximide treatment failure occurs in 55\% of patients during the first year of treatment (Glauser et al., 2013), which could be due to ethosuximide's broad mechanism of action involving the reduction of excitation (Leresche et al., 1998; Crunelli and Leresche, 2002). In order to develop safe and targeted patient-specific AEDs, there is a need to identify the different cellular and molecular mechanisms underlying absence seizures, taking into account possible variations in geneticallydifferent patients.

Data from human and animal studies have established that absence seizures are associated with spike-wave discharges (SWDs), which arise from aberrant hypersynchronous activity within the corticothalamocortical (CTC) network (Snead, 1995; McCormick and Contreras, 2001). The hypersynchronous oscillations appear to be a consequence of distinct aberrations in different microcircuits of the corticothalamic (CT) and thalamocortical (TC) nodes, which shift the balance between excitatory and inhibitory synaptic transmission in neural circuits. There appear to be multiple mechanisms through which this imbalance can occur. Altered glutamatergic excitation, mainly due to altered receptor function, has been implicated in epileptogenesis in many experimental models (Meldrum et al., 1999). In line with this, perampanel, a non-competitive antagonist of AMPA receptors (AMPARs) (Hanada et al., 2011) that mediates most of the fast glutamatergic excitatory transmission in the brain (Seeburg, 1993), is used in the treatment of some focal and generalized seizures (Kim et al., 2016; Trinka et al., 2016). However, in trials with well-characterized genetic rodent models of absence epilepsy, perampanel, as well as other AMPAR antagonists, have been found to be ineffective against absence seizures (Kamiński et al., 2001; Jakus et al., 2004; Hanada et al., 2011; Citraro et al., 2017). This could indicate a potential difference in the AMPAR-mediated excitatory mechanisms underlying the generation of absence seizures. In earlier studies using the well-established stargazer mutant mouse model of absence epilepsy, we demonstrated a selective loss of excitatory AMPARs specifically at CT synapses onto inhibitory reticular thalamic nuclei (RTN) interneurons (Barad et al., 2012). This underlies reduced AMPAR excitatory currents in these inhibitory neurons (Menuz and Nicoll, 2008). A specific loss in CT-RTN excitation, leading to impaired feed-forward inhibition of thalamic relay nuclei, has also been demonstrated in the absence epileptic Gria4 knockout mouse, which lacks the AMPAR GluA4 subunit (Paz et al., 2011). Together, these studies suggest that a failure of feed-forward inhibitory motifs, due to an impaired glutamatergic AMPAR-mediated excitatory activation of inhibitory neurons, may be key to the generation of SWDs in some absence epilepsy seizure models.

Given that SWDs appear to be initiated in the somatosensory cortex (Meeren et al., 2005; Polack et al., 2007), we turned our attention to the stargazer somatosensory cortex to determine if a similar impairment in feed-forward inhibition is present within specific cortical networks. In the cortex, stargazin, the protein mutated in stargazers (Letts et al., 1998), is selectively expressed in inhibitory neurons, predominantly in parvalbumin-containing $\left(\mathrm{PV}^{+}\right)$neurons (Maheshwari et al., 2013; Tao et al., 2013), which mediate feed-forward inhibition. We previously demonstrated that there is no change in the density of cortical $\mathrm{PV}^{+}$inhibitory neurons, but there is a decrease in the dendrite:soma ratio of GluA1/4 AMPAR expression in these $\mathrm{PV}^{+}$neurons in stargazers in comparison to non-epileptic (NE) littermates (Adotevi and Leitch, 2016), which could indicate dendritic mistrafficking of these AMPARs to the synapse of $\mathrm{PV}^{+}$neurons, and potential dysregulation of cortical feed-forward inhibition. This finding is consistent with another study, which demonstrated that a mistrafficking of GluA4-AMPARs in $\mathrm{PV}^{+}$neurons could result in cortical interneuron dysfunction, and underlie seizure exacerbation following application of NMDA receptor (NMDAR) antagonists (Maheshwari et al., 2013). These findings suggest that in the stargazer mutant mouse, a downregulation of AMPAR-excitation onto feed-forward inhibitory neurons in the CTC circuitry may underlie seizure activity. However, these studies do not specifically analyze AMPAR expression at identified synapses.

Hence, the aim of our current study was to determine the relative expression of AMPAR at synapses in epileptic stargazers compared with their non-epileptic littermates. Biochemical fractionation was performed to isolate the subcellular components of micropunched tissue from the full depth of the somatosensory cortex of stargazer and NE control littermates. The fractions were processed by western blotting for a comparative analysis of total synaptic AMPAR GluA1-4 expression. The relative expression levels of GluA1-4 at excitatory synapses onto cortical $\mathrm{PV}^{+}$interneurons, was then analyzed using double post-embedding immunogoldcytochemistry with antibodies against PV and AMPAR GluA1-4 to determine changes in AMPAR receptor numbers at synapses of $\mathrm{PV}^{+}$neurons.

\section{MATERIALS AND METHODS}

\section{Animals}

Experimental procedures were performed on 9-12 week-old male epileptic stargazer (stg/stg) and $\mathrm{NE}(+/ \mathrm{stg},+/+)$ control littermates. These mice were offspring of stargazer breeding stock obtained from the Jackson Laboratory (Bar Harbor, ME, United States), and raised at University of Otago's Animal Resource Unit. Mice were maintained on a $12 \mathrm{~h}$ light/dark cycle, with access to food and water ad libitum. Mice genotypes were confirmed by tail DNA based on guidelines of Jackson 


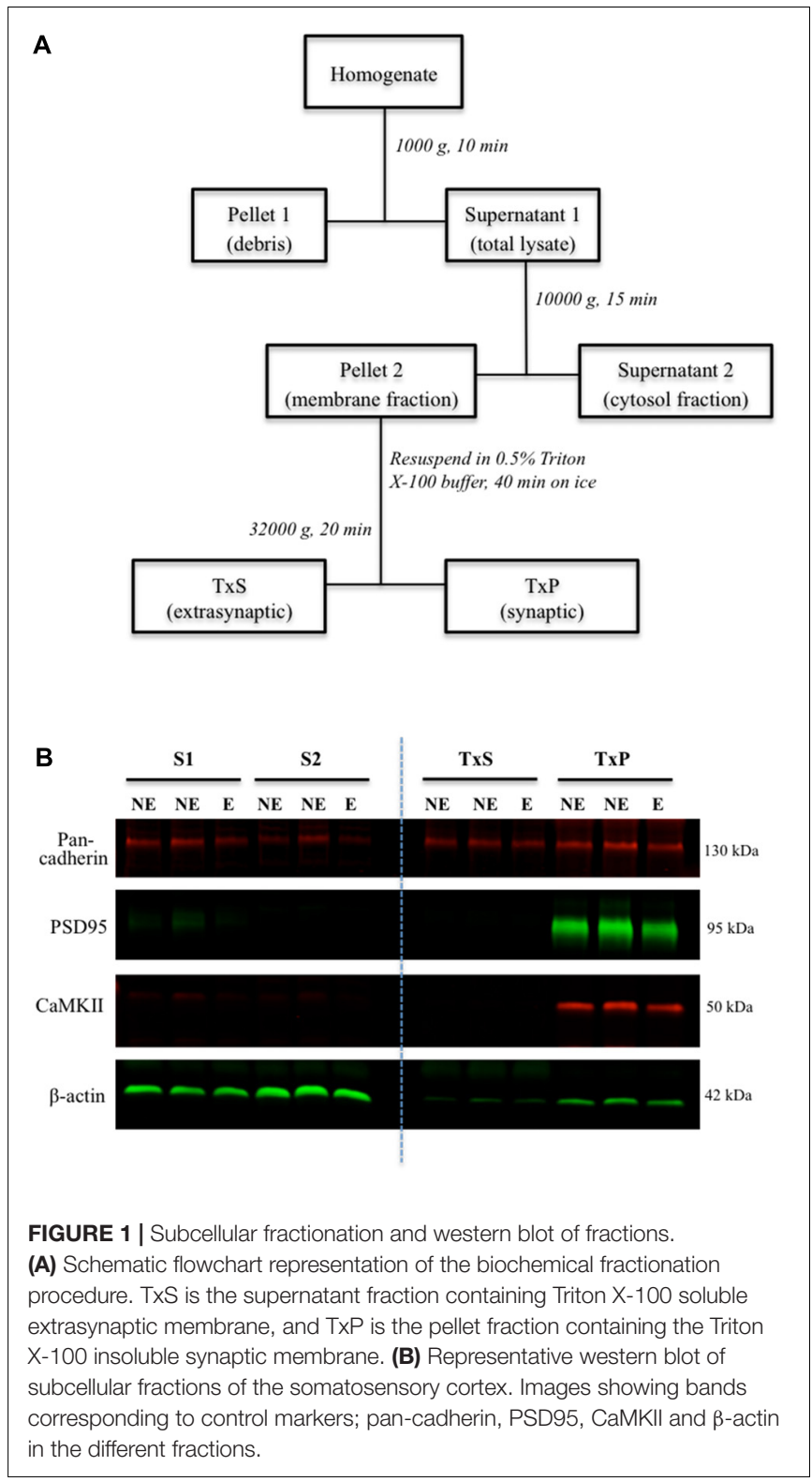

Laboratory. All experiments were carried out according to protocols approved by the University of Otago Animal Welfare Office and Ethics Committee.

\section{Biochemical Fractionation of Cortical Tissue}

Following cervical dislocation of mice, brains were extracted and snap frozen on dry ice. Brains were sectioned in a freezing cryostat (Leica CM1950, Wetzlar, Germany) into $250 \mu \mathrm{m}$ coronal sections, which were thaw-mounted onto glass slides. Micropunched tissue taken from the full depth of the somatosensory cortex was homogenized in ice-cold fractionation buffer (10 mM Tris, $320 \mathrm{mM}$ sucrose, $1 \mathrm{mM}$ EDTA, $\mathrm{pH}$ 7.4) supplemented with PMSF and protease inhibitor (Sigma, P8340), using sterile plastic pestles coupled with sonication.
Synaptic fractions were isolated using a previously described multi-step centrifugation protocol (Figure 1A) (Davies et al., 2007; Barad et al., 2017), which relies on the insolubility of synaptic membranes in Triton X-100. Briefly, homogenates were centrifuged at $1000 \mathrm{~g}$ for $10 \mathrm{~min}$ to pellet debris and yield a supernatant. The supernatant was centrifuged at $10000 \mathrm{~g}$ for $15 \mathrm{~min}$ to pellet the cell membrane (membrane fraction). The membrane fraction was resuspended in homogenization buffer containing $0.5 \%$ Triton X-100 and incubated on ice for $40 \mathrm{~min}$, and then centrifuged at $32000 \mathrm{~g}$ for $20 \mathrm{~min}$ to yield the pelleted synaptic membrane (TxP) and a supernatant containing extrasynaptic membranes $(\mathrm{TxS})$. TxP was resuspended in homogenization buffer (50 mM Tris, 2 mM EDTA, 3\% SDS, $\mathrm{pH}$ 6.8). TxS was precipitated with acetone overnight, followed by centrifugation at $3000 \mathrm{~g}$ for $15 \mathrm{~min}$, and finally resuspended in homogenization buffer for western blotting.

\section{Western Blotting of Fractions}

Western blot analysis of tissue fractions was carried out as previously described (Adotevi and Leitch, 2016). Briefly, $10 \mu \mathrm{g}$ of protein per sample was separated on $8.5 \%$ SDS-PAGE gels, and then transferred onto nitrocellulose membranes for immunoblotting. Protein expression was probed with antibodies against each of the AMPAR subunits; GluA1 (Millipore, AB1504), GluA2 (Millipore, MAB397), GluA3 (Abcam, AB40845) and GluA4 (Millipore, AB1508), as well as fraction markers; CaMKII (Santa Cruz, 13082), Pan-cadherin (Cell Signalling, 4068), PSD-95 (Synaptic Systems, 124 011), and $\beta$-actin (Abcam, AB8226). A commercially available protein standard (Novex Sharp Pre-stained Protein Standard; Life Technologies, LC5800) was included as a reference protein molecular weight ladder. Membranes were imaged with the Odyssey Imaging System (LICOR Biosciences, Lincoln, NE, United States). Relative intensities of the protein bands were measured, with normalized intensities calculated relative to the mean expression of the NE control mice.

\section{Post-embedding Immunogold Electron Microscopy}

Tissue samples were processed and analyzed according to previously established protocols from our group (Barad et al., 2012; Shevtsova and Leitch, 2012; Seo and Leitch, 2015). Mice were anesthetized with sodium pentobarbital $(60 \mathrm{mg} / \mathrm{kg}$, Pentobarb 300), and then sacrificed by transcardial perfusion with $4 \%$ paraformaldehyde and $0.1 \%$ glutaraldehyde in $0.1 \mathrm{M}$ Sorensen's phosphate buffer (PB). Brains were extracted and further post-fixed overnight at $4^{\circ} \mathrm{C}$, before sectioning into $250 \mu \mathrm{m}$ coronal sections on a vibratome (Leica VT1200, Nussloch, Germany). Tissue trimmed from the full depth of the somatosensory cortex were cryoprotected by slow infiltration in increasing concentrations of sucrose. These were then slamfrozen (Leica KF80, Vienna, Austria), followed subsequently by freeze-substitution and resin embedding (Lowicryl HM20) in an automatic freeze-substitution machine (Leica AFS). Ultrathin sections were cut and mounted onto formvar-coated nickel grids for immunolabeling. Grid sections were incubated 
in blocking buffer (10\% NGS in TBST, $\mathrm{pH}$ 7.4) for $2 \mathrm{~h}$, followed by overnight double-labeling with primary antibodies against the AMPAR GluA1, GluA2/3 (Millipore, AB1506), GluA4 subunits and parvalbumin (Swant, 235), and finally incubated in gold-conjugated to $10 \mathrm{~nm}$ goat anti-rabbit and $20 \mathrm{~nm}$ goat anti-mouse secondary antibodies (British Biocell, Cardiff, United Kingdom) to identify GluA1-4 and PV with the 10 and $20 \mathrm{~nm}$ gold particles respectively. Grids were stained with uranyl acetate and lead citrate, and imaged with the Phillips CM100 transmission electron microscope (Phillips/FEI Corporation, Eindhoven, Holland). Images were analyzed with Image J (NIH, United States), with a comparative analysis of protein expression conducted on tissue from stargazers and control NE mice processed in parallel. Excitatory synapses were identified based on their prominent post-synaptic density (PSD) and asymmetric morphology (Peters et al., 1991). The length of each labeled PSD of an identified $\mathrm{PV}^{+}$cell was measured. Gold particles located within a $30 \mathrm{~nm}$ distance from the labeled PSD of $\mathrm{PV}^{+}$neurons were regarded as synaptic, and counted as synaptic GluA1-4 subunits, for comparison between stargazer and NE mice. Density of AMPAR GluA1-4 labeling was obtained by dividing the total number of immunogold particles by the length of the PSD. Antibody specificity was confirmed by omission of primary antibodies and also by preadsorption of primary antibodies with their respective control antigens (used $5 x$ in excess), which were commercially available from the manufacturers of the primary antibodies, namely: parvalbumin recombinant for PV (Swant); GluA1 control peptide for AB1504 (Millipore, AG360); GluA2/3 control peptide for AB1506 (Millipore, AG305); and GluA4 control peptide for AB1508 (Millipore, AG306). Imaging and data analyses were performed with the experimenter blinded to the genotypes. EM grids were coded by an independent non-experimenter, with genotypes revealed only after completion of all quantitative analyses.

\section{Data Analysis}

Data are presented as mean \pm standard error of the mean (SEM). Statistical tests were performed using GraphPad Prism 7.0 (GraphPad Software, United States). Comparative analysis of differences in AMPAR expression levels between stargazers and their NE control littermates were assessed by unpaired Mann-Whitney $U$-test, with statistical significance set at $p<0.05$.

\section{RESULTS}

\section{Reduced AMPAR GluA1-4 Expression in Stargazer Cortical Synaptic Fractions}

To analyze relative expression of AMPARs at synapses in epileptic stargazers compared to NE littermates, somatosensory cortex tissue was separated into subcellular components by biochemical fractionation (Figure 1A). First, we validated the methodology by confirming the purity of the isolated fractions using western blotting and immunolabeling for synaptic specific protein markers including CaMKII and PSD-95, which are components of the PSD (Petersen et al., 2003). We also immunolabeled cell adhesion protein pan-cadherin (Beesley et al., 1995) and the cytoplasmic protein actin (Schubert and Dotti, 2007). Immunopositive bands corresponding to the markers were detected at their manufacturer-specified molecular weights (42, 50, 95, and $130 \mathrm{kDA}$ respectively). As expected, PSD95 and CaMKII, were enriched in synaptic fractions (TxP), with very low levels in the total lysate (S1). $\beta$-actin was present in all fractions except the extrasynaptic membrane (TxS), whereas pan-cadherin was detected in all fractions (Figure 1B). To quantify synaptic AMPAR expression in our fractions, we immunolabeled western blots with antibodies against GluA14 subunits, which are all expressed in the somatosensory cortex (Adotevi and Leitch, 2016). GluA1-4 expression was enriched in synaptic fractions (TxP), with detectable, but low levels in the extrasynaptic fractions (TxS; Figures 2A-D). Because neuronal cadherin does not associate with stargazin (Tomita et al., 2003), and its expression is unaltered in stargazers compared to NE controls (Barad et al., 2017), the AMPAR GluA1-4 relative intensities were normalized to their corresponding pan-cadherin protein band. Analysis of relative AMPAR subunit expression levels in TxP fractions showed that expression of all GluA1-4 subunits was significantly decreased in stargazers compared to their NE littermates. Relative synaptic expression of GluA1 was decreased by $21 \%(n=6, p<0.05$, Figures 2A,E), 23\% in GluA2 $(n=5, p<0.05$, Figures 2B,E), $23 \%$ in GluA3 ( $n=7, p<0.05$, Figures 2C,E), and $34 \%$ in GluA4 $(n=5, p<0.01$, Figures 2D,E) in epileptic stargazers compared to NE littermates. Full length western blots and Supplementary Material are provided in Supplementary Figures 1-3.

\section{Loss of AMPAR Expression at Excitatory Synapses onto Cortical PV ${ }^{+}$Neurons}

Having established that AMPARs were significantly reduced in synaptic fractions (TxP), of stargazers compared to $\mathrm{NE}$ controls we next wanted to determine if there was a specific loss of AMPARs at synaptic inputs to inhibitory feed-forward neurons in the cortex. We had previously found a loss in the dendrite:soma ratio of GluA1/4-AMPARs in stargazer $\mathrm{PV}^{+}$neurons (Adotevi and Leitch, 2016), which could be an indication of loss of AMPAR-mediated excitation of these neurons. To determine if these changes in AMPAR expression were evident at excitatory synapses onto these $\mathrm{PV}^{+}$neurons, the distribution of GluA1-4 immunogold particles associated with PSDs of these synapses were analyzed in stargazer and control NE mice ( $n=5$ pairs). For each AMPAR subunit, a total of 200 labeled excitatory synapses of immunopositive $\mathrm{PV}^{+}$neurons per subunit per mice group were analyzed. The identification of cortical $\mathrm{PV}^{+}$interneurons was based on the observation of parvalbumin labeling $(20 \mathrm{~nm}$ gold particles, red asterisk, Figure 3A) as described in other studies (Pelkey et al., 2015; Yamasaki et al., 2016). Excitatory synapses onto these $\mathrm{PV}^{+}$neurons were identified by their asymmetrical PSD, as well as, AMPAR immunogold $(10 \mathrm{~nm}$ ) labeling (red arrows, Figure 3A). The low levels of background labeling in omission (Figure 3B) and preadsorption (Figure 3C) control tissue 


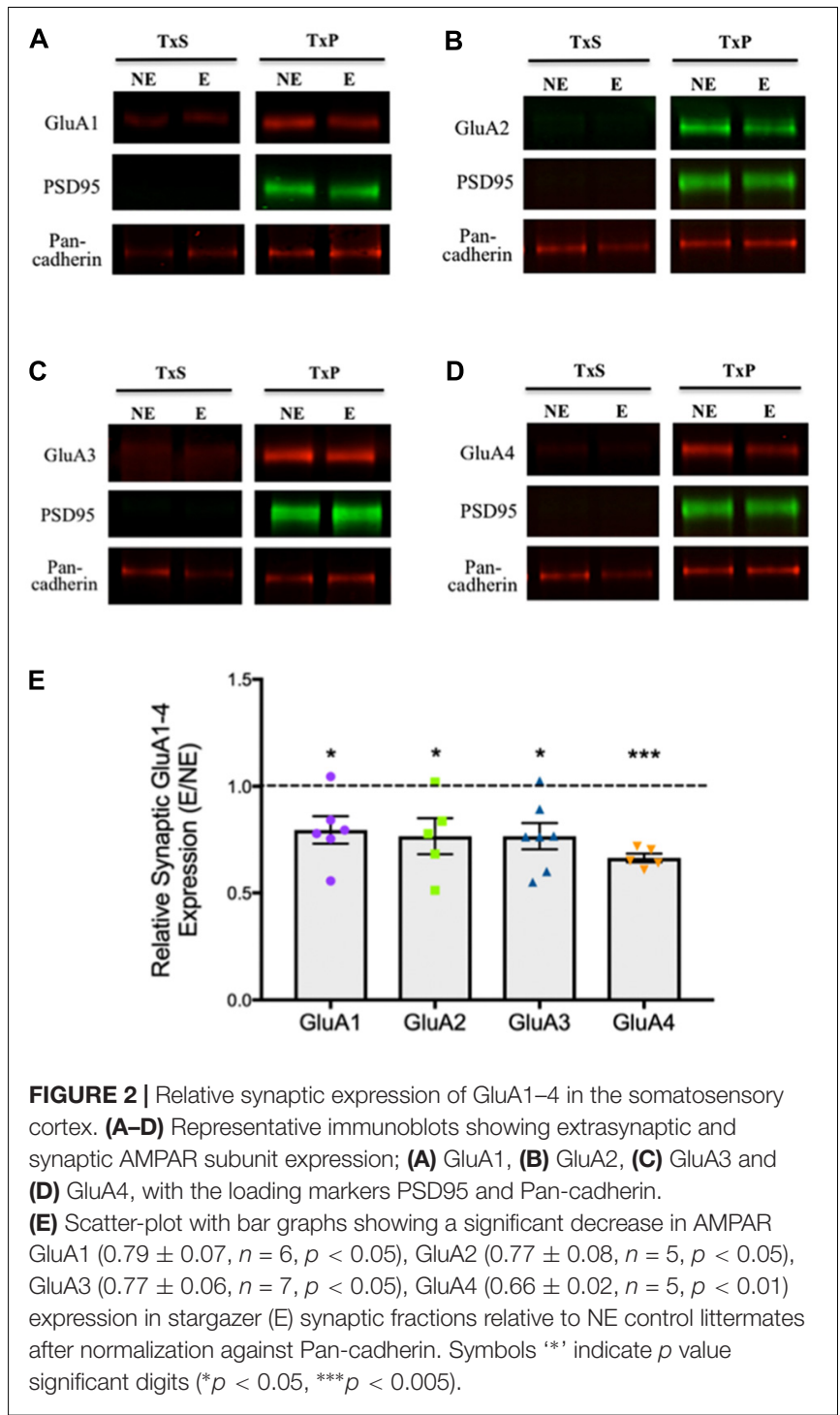

confirmed the specificity of the immunogold labeling observed in this study.

Quantitative analysis of the relative density of synaptic AMPAR subunits (gold particles per $\mu \mathrm{m}$ PSD length) in epileptic and NE littermates revealed statistically significant decreases in GluA1-4 expression at excitatory synapses of stargazer $\mathrm{PV}^{+}$ neurons compared to NE mice (Figure 4), consistent with previous observations of alterations in total synaptic expression, which could indicate possible defects in AMPAR trafficking. GluA1 expression was reduced by $21 \%$ (NE $6.98 \pm 0.306$, E $5.54 \pm 0.264, p<0.0001$; Figures $4 \mathrm{~A}, \mathrm{~B}$ ), GluA2/3 by $18 \%$ (NE $7.38 \pm 0.320$, E $6.06 \pm 0.240, p<0.005$; Figures 4C,D) and GluA4 by $29 \%$ (NE $7.42 \pm 0.344$, E $5.25 \pm 0.210, p<0.0001$; Figures $4 E, F)$. There was no statistical difference in average PSD length of excitatory synapses between stargazers and their NE littermates in the somatosensory cortex (NE $322.7 \pm 5.235 \mathrm{~nm}$, E $318.0 \pm 5.317 \mathrm{~nm}, p=0.5297$ ), which is in agreement with previous studies in the stargazer cerebellum (Richardson and
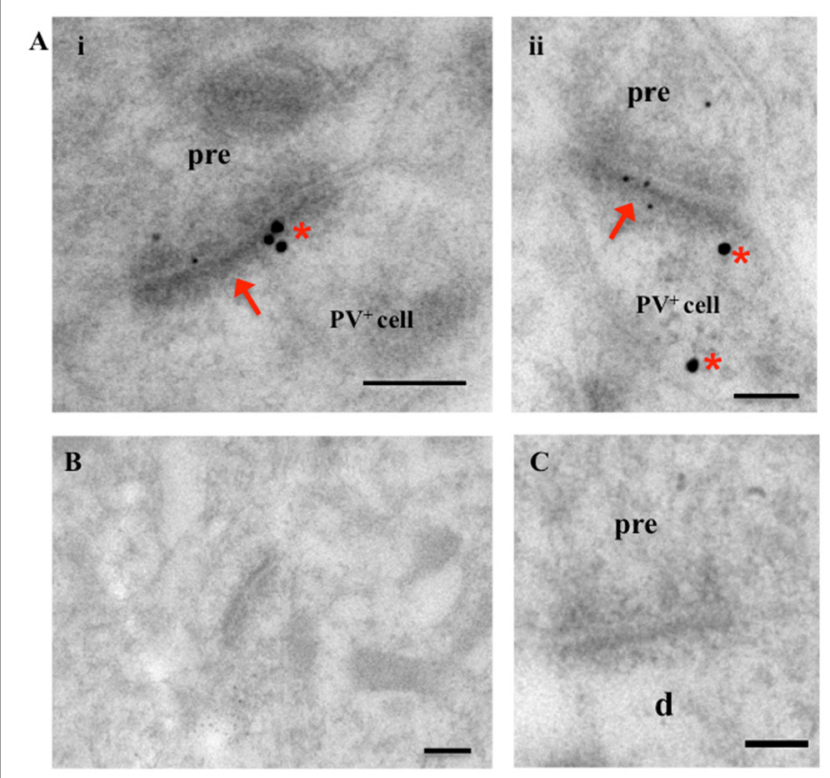

FIGURE 3 | Electron micrographs of excitatory synapses onto PV+ interneurons in the somatosensory cortex. (A) Images showing excitatory synapses (red arrows) onto $\mathrm{PV}^{+}$neurons (labeled with $20 \mathrm{~nm}$ immunogold particles; identified by red asterisk). Synapses show AMPAR subunit labeling (smaller, $10 \mathrm{~nm}$ gold particles). (B,C) Micrographs of cortical tissue with omission (B) and pre-adsorption (C) of primary AMPAR and PV antibodies as controls. Absence of gold particles in EM images indicates specificity of immunolabeling in this study. Scale bars $=200 \mathrm{~nm}$.

Leitch, 2005; Meng et al., 2006). In the current study, an antibody that recognizes both GluA2 and GluA3 (anti-GluA2/3; Millipore, AB1506) was used to label synapses immunopositive for GluA2 or GluA3, as immunolabeling with commercial antibodies against either GluA2 or GluA3, which work well in western blot applications, was unsuccessful in electron microscopy (EM) immunogold-cytochemistry applications. Hence, the quantified gold particles represent both subunits. Nonetheless, the similarity in total percentage reductions in total synaptic GluA2 and GluA3 expression, suggest that GluA2 and 3 are decreased in similar proportions at excitatory synapses of stargazer $\mathrm{PV}^{+}$neurons. Notably, previous studies have also reported that GluA2 and GluA3 cortical expression patterns are similar (Sato et al., 1993; Kondo et al., 1997), with GluA3 mostly associated with GluA2 to form GluA2/3 heteromers (Lu et al., 2009).

To determine if the changes in synaptic AMPAR subunit expression were specific to $\mathrm{PV}^{+}$interneurons, quantitative immunogold analysis of the main AMPAR subunits (GluA1-3) expressed by parvalbumin-negative (PV-) neurons (Adotevi and Leitch, 2016) was conducted (Figure 5). In contrast to observed reduction of AMPAR expression at excitatory synaptic inputs onto $\mathrm{PV}^{+}$interneurons in stargazers, there was no statistical difference in the relative densities of AMPAR at excitatory synapses in PV negative neurons in stargazers compared to their NE littermates (GluA1: NE $6.71 \pm 0.339$, E $6.60 \pm 0.336$, $p=0.6156$, Figures 5A,B and GluA2/3: NE $6.30 \pm 0.349, \mathrm{E}$ $6.22 \pm 0.317, p=0.7749$; Figures 5 C,D). These data provide 
A

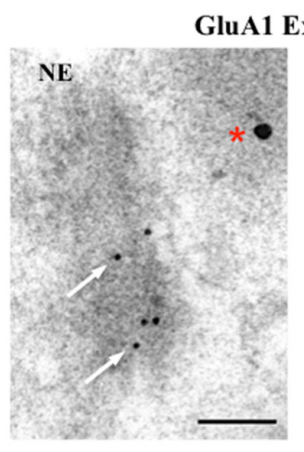

C

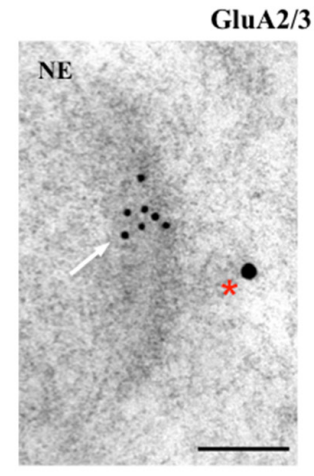

GluA2/3 Expression

E

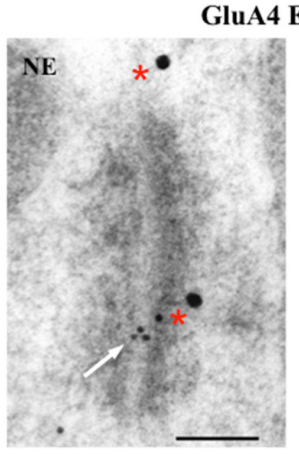

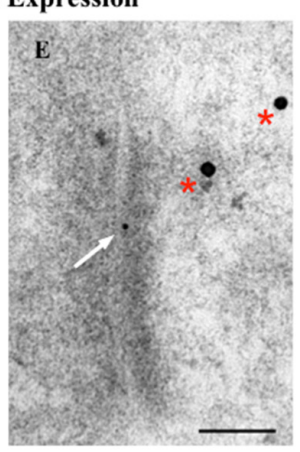
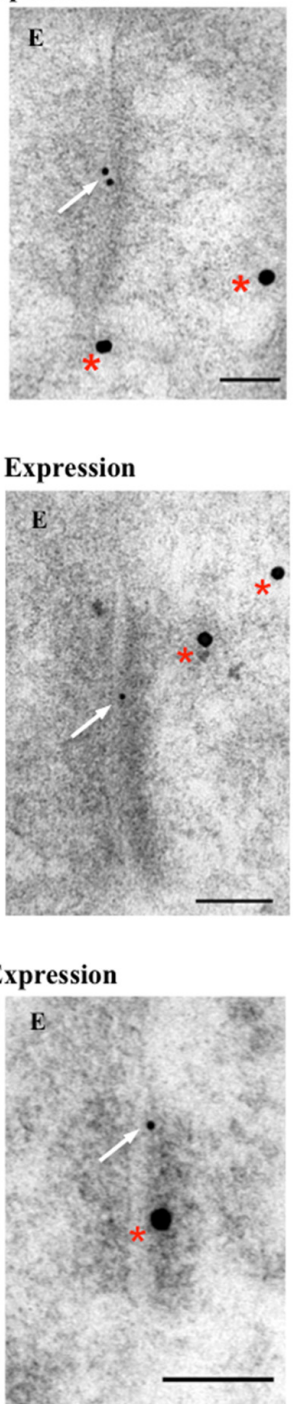

B

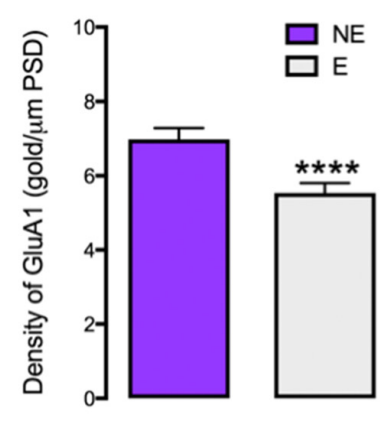

D

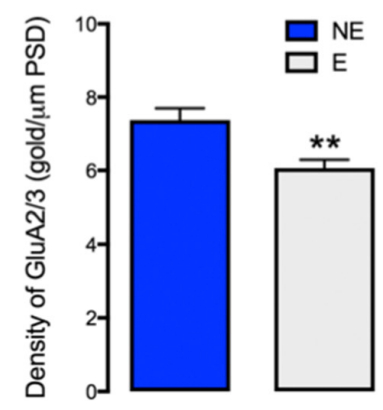

$\mathbf{F}$

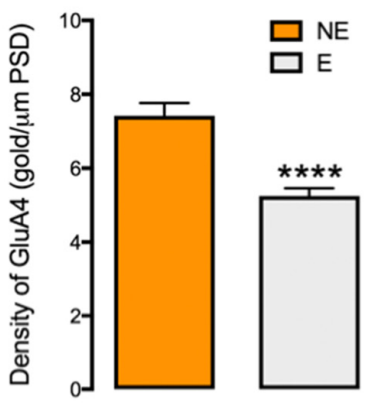

FIGURE 4 | GluA1-4 immunogold labeling at excitatory synapses onto cortical PV+ neurons (A,C,E) EM micrographs showing labeling of GluA1 (A), GluA2/3 (C), and GluA4 (E) at excitatory synapses onto $\mathrm{PV}^{+}$neurons in the somatosensory cortex of NE control and stargazer (E) mice. Off-white arrows indicate gold particles representative of AMPAR subunits' labeling whereas red asterisks are indicative of PV labeling. (B,D,F) Quantitative analysis of GluA1-4 immunogold density per $\mu \mathrm{m}$ PSD length shows reduced GluA1 (NE $6.98 \pm 0.306, E 5.54 \pm 0.264, p<0.0001$ ), GluA2/3 (NE $7.38 \pm 0.320, E 6.06 \pm 0.240, p<0.005$ ) and GluA4 (NE $7.42 \pm 0.344$, E $5.25 \pm 0.210, p<0.0001$ ) expression in stargazers (E) compared to control NE mice ( $n=5$ pairs). Scale bars $=100 \mathrm{~nm}$. Symbols '*’ indicate $p$ value significant digits $\left(* * p<0.005,{ }^{* * * *} p<0.0001\right)$.

evidence that the reduction in AMPAR expression at synapses in the somatosensory cortex of stargazers affects $\mathrm{PV}^{+}$neurons.

\section{DISCUSSION}

In the current study, we analyzed the synaptic expression of AMPAR GluA1-4 subunits in the somatosensory cortex of stargazers compared to their NE control littermates to determine if there is a loss of AMPAR expression at input synapses onto inhibitory interneurons, based on our previous findings suggestive of a dendritic mistrafficking of AMPARs in cortical
$\mathrm{PV}^{+}$neurons (Adotevi and Leitch, 2016). We demonstrate for the first time that, in the stargazer cortex there is a reduction of total synaptic levels of GluA1-4 in isolated synaptic fractions analyzed with western blotting, and from EM immunogold analysis, a loss of these AMPAR GluA1-4 subunits at excitatory synapses onto inhibitory $\mathrm{PV}^{+}$neurons. These findings provide evidence of a potential loss of synaptic AMPAR-mediated excitation of cortical inhibitory neuron, which could consequently weaken feed-forward inhibitory output, and underlie the generation of SWDs in stargazers.

The loss of GluA1 and 4 containing AMPARs at $\mathrm{PV}^{+}$ neuron synapses is not surprising, given that studies report that 
A

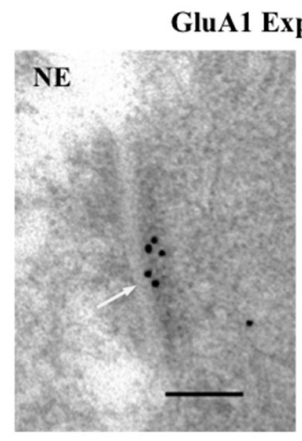

C

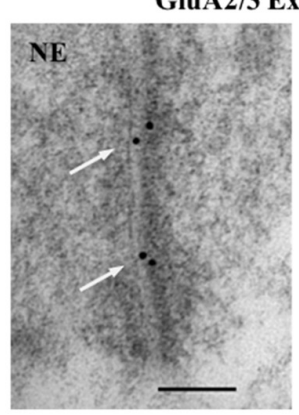

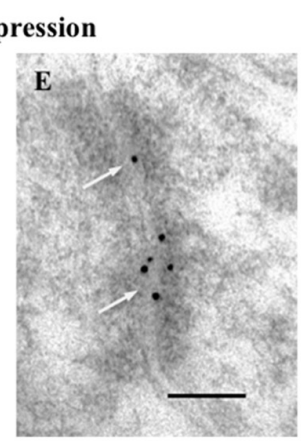

pression

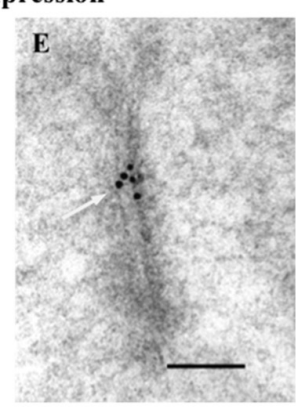

B

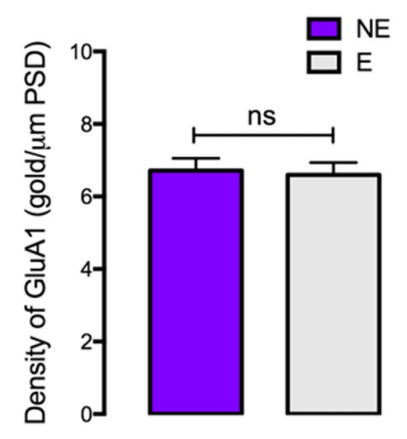

D

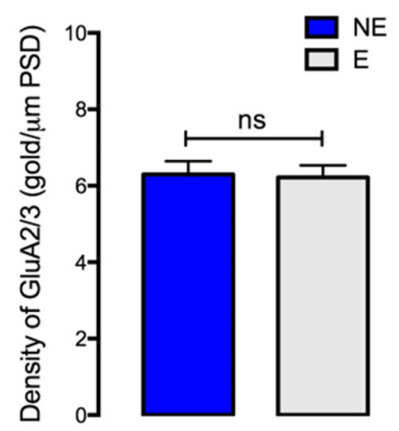

FIGURE 5 | GluA1-3 immunogold labeling at excitatory synapses onto cortical PV negative neurons (A,C) EM micrographs showing labeling of GluA1 (A) and GluA2/3 (C) at excitatory synapses onto $\mathrm{PV}^{-}$neurons in the somatosensory cortex of NE control and stargazer (E) mice. White arrows indicate gold particles representative of AMPAR subunits' labeling. (B,D) Quantitative analysis of GluA1-3 immunogold density per $\mu \mathrm{m}$ PSD length showed no differences in GluA1 (NE $6.71 \pm 0.339, \mathrm{E} 6.60 \pm 0.336, p=0.6156$ ) and GluA2/3 (NE $6.30 \pm 0.349, \mathrm{E} 6.22 \pm 0.317, p=0.7749$ ) expression between stargazers (E) and control NE mice $(n=5$ pairs). Scale bars $=100 \mathrm{~nm}$.

$\mathrm{PV}^{+}$neurons predominantly express GluA1 and 4-containing AMPARs (Geiger et al., 1995; Kwok et al., 1997). Furthermore, it has been previously reported that stargazin expression is associated with GluA4 containing $\mathrm{PV}^{+}$neurons in the stargazer cortex (Maheshwari et al., 2013). Given that most cortical GluA4 $^{+}$neurons co-express GluA1 (Kondo et al., 1997), this may also account for the 29 and 21\% reductions in GluA4 and GluA1 expression respectively at $\mathrm{PV}^{+}$synapses. Nonetheless, the effects of the stargazin deficit does not seem limited to only GluA1/4-AMPARs, as we also observed a reduction of GluA2/3 expression (GluA2/3 expression was reduced in total synaptic fractions by $23 \%$ and at identified $\mathrm{PV}^{+}$synapses by $\left.18 \%\right)$. These subunits are also expressed in $\mathrm{PV}^{+}$neurons (Geiger et al., 1995; Kwok et al., 1997; Wang and Gao, 2010). These results are consistent with findings in the stargazer thalamus, which also shows a loss of GluA2/3-AMPARs at inhibitory RTN synapses (Barad et al., 2012). Thus, taken together, our results indicate a significant loss of AMPARs, predominantly GluA4-containing AMPARs, at synapses onto $\mathrm{PV}^{+}$neurons in the stargazer somatosensory cortex.

Although we observed a significant reduction in AMPAR expression, a proportion of receptors were still present at synapses of stargazer $\mathrm{PV}^{+}$neurons. Other studies on stargazers indicate that total absence of GluA subunits only occurs at synapses of neurons (e.g., granule cells of the cerebellum), which depend exclusively on stargazin as their sole Type I TARP (Chen et al., 2000; Tomita et al., 2003; Fukaya et al., 2005; Vandenberghe et al., 2005). Conversely, in the stargazer thalamus (Barad et al., 2012) and cortex where there is reduced but not complete loss of synaptic AMPARs, this may indicate that other TARPs or alternatively other AMPAR auxiliary proteins such cornichons (Schwenk et al., 2009) could mediate AMPAR trafficking to these synapses in the absence of stargazin. Nonetheless, our results indicate a marked reduction in AMPAR expression at $\mathrm{PV}^{+}$interneuron synapses, which suggests that the potential expression of other auxiliary proteins does not fully compensate for the loss of stargazin in these neurons. Additionally, our data indicate that the loss of AMPAR expression in the stargazer cortex affects $\mathrm{PV}^{+}$neurons since no changes in subunit expression were observed at synaptic inputs onto $\mathrm{PV}^{-}$neurons (other non-parvalbumin inhibitory neurons and excitatory neurons). Hence, our current findings give credence to the proposal that reduced AMPAR-mediated excitation of fast-spiking $\mathrm{PV}^{+}$ neurons engenders a subsequent failure of these inhibitory neurons to provide adequate feed-forward inhibition within cortical networks.

The loss of feed-forward inhibition within the CTC circuitry, which is required to prevent runaway excitation, has been suggested as a potential SWD-causing mechanism in different models of absence epilepsy (Sasaki et al., 2006; Paz et al., 2011; Maheshwari et al., 2013; Adotevi and Leitch, 2016). Recent evidence indicates that the loss of excitatory input, 
particularly to inhibitory $\mathrm{PV}^{+}$interneurons (Maheshwari et al., 2013; Pelkey et al., 2015; Adotevi and Leitch, 2016), could affect inhibitory feed-forward activity and thus render the CTC circuitry susceptible to SWDs. AMPARs primarily drive fast and robust excitatory neurotransmission in the CNS, and previous studies have shown that excitatory inputs onto inhibitory interneurons are often larger in magnitude than equivalent inputs onto neighboring excitatory pyramidal cells (Porter et al., 2001; Gabernet et al., 2005; Cruikshank et al., 2007). $\mathrm{PV}^{+}$neurons depress rapidly once activated by excitatory inputs to mediate "early-onset" feed-forward inhibition of excitatory pyramidal cells, which shortens the temporal "window" for excitation (Lalanne et al., 2016), and thus prevents overexcitation within cortical networks. Indeed, seizures are thought to be a result of neuronal hyperexcitability, due to an imbalance between excitatory and inhibitory transmission (Snead, 1995; Timofeev and Steriade, 2004). Given that in the cortex, stargazin is predominantly expressed in $\mathrm{PV}^{+}$inhibitory interneurons, it is most likely that excitation of pyramidal neurons remains intact. This is further supported by our finding that there is no loss of AMPAR expression at PV negative neurons in the somatosensory cortex, of which the majority are likely to be pyramidal cells. Hence, the loss of GluA1-4 AMPARs in stargazer $\mathrm{PV}^{+}$ neurons, demonstrated in this study, could lead to a dysfunction of cortical $\mathrm{PV}^{+}$feed-forward inhibitory microcircuits due to reduced excitation-induced activation of these interneurons. This is in agreement with the suggestion that a reduction of synaptic excitation of $\mathrm{PV}^{+}$neurons, but not excitatory neurons, may result in a decrease in feed-forward inhibition ( $\mathrm{Paz}$ and Huguenard, 2015). The impaired feed-forward inhibition would be insufficient to overcome the recurrent excitation of cortical pyramidal neurons (Douglas et al., 1995), and thus create a hyperexcitable cortex susceptible to SWDs.

Although other researchers have proposed an alternative mechanism wherein NMDARs are capable of mediating the excitation of inhibitory neurons in the absence of AMPARs, and even upregulate inhibitory function (Lacey et al., 2012), studies of these NMDARs in stargazer CTC oscillatory activity continue to provide contradictory results (Aizawa et al., 1997; Lacey et al., 2012; Maheshwari et al., 2013; Barad et al., 2017). For instance, in a very recent study, even in the absence of synaptic AMPAR expression in the stargazer RTN, there was no corresponding compensatory increase in synaptic NMDAR expression (Barad et al., 2017). In addition, all the conclusions from the different studies do suggest that changes in NMDAR expression and function in inhibitory networks, if any, are a consequence of preceding changes in AMPAR expression and function, highlighting a critical role for the loss of AMPAR-mediated activation of $\mathrm{PV}^{+}$ feed-forward interneurons in SWDs in stargazers. Another mechanism for a reduction in excitatory drive onto PV neurons could be a reduction in the number of excitatory synapses onto these neurons (as occurs in schizophrenia, Chung et al., 2016). Although, a quantification of excitatory synapse numbers onto PV neurons was not conducted in this study, it is possible that the stargazer mutation also impacts on synapse density.
In summary, the loss of synaptic AMPAR-mediated excitation of $\mathrm{PV}^{+}$neurons suggests that disinhibition in cortical networks, due to insufficient feed-forward inhibition of excitatory neurons, may underlie seizure activity in the stargazer mutant mouse. In this way, impaired feed-forward inhibition seems to be a SWD-generating factor in some cases of absence epilepsy. Of note, stargazin expression has also been reported in a smaller number of other cortical GABAergic inhibitory interneurons, e.g., somatostatin-expressing $\left(\mathrm{SOM}^{+}\right)$neurons (Tao et al., 2013). Hence, it is possible that AMPAR expression may also be altered in these interneurons, and thus epileptic activity in stargazer is associated with a reduction in all types of GABAergic inhibition. Given that feed-forward inhibition is primarily mediated by $\mathrm{PV}^{+}$neurons (Freund and Katona, 2007; Paz and Huguenard, 2015), future studies using methodologies such as DREADDs technology to selectively manipulate the action of cortical inhibitory interneurons could elucidate the dynamics of feedforward inhibition in absence seizures. And thus, substantiate the proposal that the specific targeting and regulation of excitatory activation of inhibitory interneurons could be a potential seizuresuppressing mechanism in some absence epilepsy patients.

\section{AUTHOR CONTRIBUTIONS}

BL conception, hypothesis development and design of the research, secured the funding. NA helped with research design and conducted experiments. Analyses, interpretation of the results were conducted jointly. Both authors contributed to manuscript writing and approved the final version.

\section{FUNDING}

We also acknowledge the University of Otago for the Doctoral Scholarship awarded to NA. This work was supported by grants from the University of Otago Research Grants (UORG) and Deans Bequest Fund awarded to BL.

\section{ACKNOWLEDGMENTS}

The authors thank the staff of the Otago Centre for Electron Microscopy (OCEM) and Rob Alumbaugh (Pharmacology and Toxicology, University of Otago) for technical support.

\section{SUPPLEMENTARY MATERIAL}

The Supplementary Material for this article can be found online at: https://www.frontiersin.org/articles/10.3389/fnmol.2017. 00434/full\#supplementary-material

FIGURE S1 | Western blot analysis of subcellular isolated fractions of the mouse somatosensory cortex. Blot images showing bands corresponding to control markers; pan-cadherin, PSD95, CaMKII and $\beta$-actin in the different fractions. $\mathrm{S} 1$ is the supernatant 1 containing total cortical tissue lysate, S2 is the supernatant containing the cytosol fraction, TxS is the supernatant fraction containing Triton 
$\mathrm{X}-100$ soluble extrasynaptic membrane, and TXP is the pellet fraction containing the Triton X-100 insoluble synaptic membrane.

FIGURE S2 | Western blot analysis of GluA1-4 expression in extrasynaptic (TxS) and synaptic (TxP) fractions. Images showing expression of (A) GluA1, (B) GluA2 and GluA4, (C) GluA3 and the loading control markers pan-cadherin and PSD95.

\section{REFERENCES}

Adotevi, N. K., and Leitch, B. (2016). Alterations in AMPA receptor subunit expression in cortical inhibitory interneurons in the epileptic stargazer mutant mouse. Neuroscience 339, 124-138. doi: 10.1016/j.neuroscience.2016.09.052

Aizawa, M., Ito, Y., and Fukuda, H. (1997). Pharmacological profiles of generalized absence seizures in lethargic, stargazer and gamma hydroxybutyrate- treated model mice. Neurosci. Res. 29, 17-25. doi: 10.1016/S0168-0102(97)00066-7

Barad, Z., Grattan, D. R., and Leitch, B. (2017). NMDA receptor expression in the thalamus of the stargazer model of absence epilepsy. Sci. Rep. 7:42926. doi: $10.1038 /$ srep42926

Barad, Z., Shevtsova, O., Arbuthnott, G. W., and Leitch, B. (2012). Selective loss of AMPA receptors at corticothalamic synapses in the epileptic stargazer mouse. Neuroscience 217, 19-31. doi: 10.1016/j.neuroscience.2012.05.011

Beesley, P. W., Mummery, R., and Tibaldi, J. (1995). N-cadherin is a major glycoprotein component of isolated rat forebrain postsynaptic densities. J. Neurochem. 64, 2288-2294. doi: 10.1046/j.1471-4159.1995.64052288.x

Berg, A. T., Berkovic, S. F., Brodie, M. J., Buchhalter, J., Cross, J. H., van Emde Boas, W., et al. (2010). Revised terminology and concepts for organization of seizures and epilepsies: report of the ILAE commission on classification and terminology, 2005-2009. Epilepsia 51, 676-685. doi: 10.1111/j.1528-1167.2010. 02522

Caplan, R., Siddarth, P., Stahl, L., Lanphier, E., Vona, P., Gurbani, S., et al. (2008). Childhood absence epilepsy: behavioral, cognitive, and linguistic comorbidities. Epilepsia 49, 1838-1846. doi: 10.1111/j.1528-1167.2008.01680

Chen, L., Chetkovich, D. M., Petralia, R. S., Sweeney, N. T., Kawasaki, Y., Wenthold, R. J., et al. (2000). Stargazin regulates synaptic targeting of AMPA receptors by two distinct mechanisms. Nature 408, 936-943. doi: 10.1038/ 35050030

Chung, D. W., Fish, K. N., and Lewis, D. A. (2016). Pathological basis for deficient excitatory drive to cortical parvalbumin interneurons in schizophrenia. Am. J. Psychiatry 173, 1131-1139. doi: 10.1176/appi.ajp.2016.16010025

Citraro, R., Leo, A., Franco, V., Marchiselli, R., Perucca, E., De Sarro, G., et al. (2017). Perampanel effects in the WAG/Rij rat model of epileptogenesis, absence epilepsy, and comorbid depressive-like behavior. Epilepsia 58, 231-238. doi: 10.1111/epi.13629

Cruikshank, S. J., Lewis, T. J., and Connors, B. W. (2007). Synaptic basis for intense thalamocortical activation of feedforward inhibitory cells in neocortex. Nat. Neurosci. 10, 462-468. doi: 10.1038/nn1861

Crunelli, V., and Leresche, N. (2002). Block of thalamic T-Type Ca2+ channels by ethosuximide is not the whole story. Epilepsy Curr. 2, 53-56. doi: 10.1046/j. 1535-7597.2002.00024

Davies, K. D., Alvestad, R. M., Coultrap, S. J., and Browning, M. D. (2007). alphaCaMKII autophosphorylation levels differ depending on subcellular localization. Brain Res. 1158, 39-49. doi: 10.1016/j.brainres.2007.05.008

Douglas, R. J., Koch, C., Mahowald, M., Martin, K. A., and Suarez, H. H. (1995). Recurrent excitation in neocortical circuits. Science 269, 981-985. doi: 10.1126/ science.7638624

Freund, T. F., and Katona, I. (2007). Perisomatic inhibition. Neuron 56, 33-42. doi: 10.1016/j.neuron.2007.09.012

Fukaya, M., Yamazaki, M., Sakimura, K., and Watanabe, M. (2005). Spatial diversity in gene expression for VDCC $\gamma$ subunit family in developing and adult mouse brains. Neurosci. Res. 53, 376-383. doi: 10.1016/j.neures.2005.08.009

Gabernet, L., Jadhav, S. P., Feldman, D. E., Carandini, M., and Scanziani, M. (2005). Somatosensory integration controlled by dynamic thalamocortical feedforward inhibition. Neuron 48, 315-327. doi: 10.1016/j.neuron.2005.09.022

Geiger, J. R. P., Melcher, T., Koh, D. S., Sakmann, B., Seeburg, P. H., Jonas, P., et al. (1995). Relative abundance of subunit mRNAs determines gating and Ca2+ permeability of AMPA receptors in principal neurons and interneurons in rat CNS. Neuron 15, 193-204. doi: 10.1016/0896-6273(95)90076-4
FIGURE S3 | Western blot analysis of CaMKII expression in synaptic fractions. (A) Blot images showing expression of CaMKII, PSD95 and Pan-cadherin in isolated subcellular fractions. (B) Bar graph showing no difference in CaMKII expression in synaptic (TxP) fractions (highlighted in yellow) in stargazers compared to NE controls after normalization with pan-cadherin (NE 1.00 \pm 0.030 , $\mathrm{E} 1.05 \pm 0.1176, p=0.1436)$.

Glauser, T. A., Cnaan, A., Shinnar, S., Hirtz, D. G., Dlugos, D., Masur, D., et al. (2013). Ethosuximide, valproic acid, and lamotrigine in childhood absence epilepsy: initial monotherapy outcomes at 12 months. Epilepsia 54, 141-155. doi: 10.1111/epi.12028

Goren, M. Z., and Onat, F. (2007). Ethosuximide: from bench to bedside. CNS Drug Rev. 13, 224-239. doi: 10.1111/j.1527-3458.2007.00009

Hanada, T., Hashizume, Y., Tokuhara, N., Takenaka, O., Kohmura, N., Ogasawara, A., et al. (2011). Perampanel: a novel, orally active, noncompetitive AMPA-receptor antagonist that reduces seizure activity in rodent models of epilepsy. Epilepsia 52, 1331-1340. doi: 10.1111/j.1528-1167.2011.03109

Jakus, R., Graf, M., Ando, R. D., Balogh, B., Gacsalyi, I., Levay, G., et al. (2004). Effect of two noncompetitive AMPA receptor antagonists GYKI 52466 and GYKI 53405 on vigilance, behavior and spike-wave discharges in a genetic rat model of absence epilepsy. Brain Res. 1008, 236-244. doi: 10.1016/j.brainres. 2004.01.087

Kamiński, R. M., Van Rijn, C. M., Turski, W. A., Czuczwar, S. J., and Van Luijtelaar, G. (2001). AMPA and GABAB receptor antagonists and their interaction in rats with a genetic form of absence epilepsy. Eur. J. Pharmacol. 430, 251-259. doi: 10.1016/S0014-2999(01)01393-0

Kim, H. D., Chi, C. S., Desudchit, T., Nikanorova, M., Visudtibhan, A., Nabangchang, C., et al. (2016). Review of clinical studies of perampanel in adolescent patients. Brain Behav. 6:e00505. doi: 10.1002/brb3.505

Kondo, M., Sumino, R., and Okado, H. (1997). Combinations of AMPA receptor subunit expression in individual cortical neurons correlate with expression of specific calcium-binding proteins. J. Neurosci. 17, 1570-1581.

Kwok, K. H. H., Tse, Y. C., Wong, R. N. S., and Yung, K. K. L. (1997). Cellular localization of GluR1, GluR2/3 and GluR4 glutamate receptor subunits in the neurons of the rat neostriatum. Brain Res. 778, 43-55. doi: 10.1016/S00068993(97)00950-5

Lacey, C. J., Bryant, A., Brill, J., and Huguenard, J. R. (2012). Enhanced NMDA receptor-dependent thalamic excitation and network oscillations in stargazer mice. J. Neurosci. 32, 11067-11081. doi: 10.1523/jneurosci.5604-11.2012

Lalanne, T., Oyrer, J., Mancino, A., Gregor, E., Chung, A., Huynh, L., et al. (2016). Synapse-specific expression of calcium-permeable AMPA receptors in neocortical layer 5. J. Physiol. 594, 837-861. doi: 10.1113/jp271394

Leresche, N., Parri, H. R., Erdemli, G., Guyon, A., Turner, J. P., Williams, S. R., et al. (1998). On the action of the anti-absence drug ethosuximide in the rat and cat thalamus. J. Neurosci. 18, 4842-4853.

Letts, V. A., Felix, R., Biddlecome, G. H., Arikkath, J., Mahaffey, C. L. Valenzuela, A., et al. (1998). The mouse stargazer gene encodes a neuronal Ca2+-channel $\gamma$ subunit. Nat. Genet. 19, 340-347. doi: 10.1038/1228

Loscher, W. (2017). Animal models of seizures and epilepsy: Past, present, and future role for the discovery of antiseizure drugs. Neurochem. Res. 42, 1873-1888. doi: 10.1007/s11064-017-2222

Lu, W., Shi, Y., Jackson, A. C., Bjorgan, K., During, M. J., Sprengel, R., et al. (2009). Subunit composition of synaptic AMPA receptors revealed by a single-cell genetic approach. Neuron 62, 254-268. doi: 10.1016/j.neuron.2009.02.027

Maheshwari, A., Nahm, W. K., and Noebels, J. L. (2013). Paradoxical proepileptic response to NMDA receptor blockade linked to cortical interneuron defect in stargazer mice. Front. Cell. Neurosci. 7:156. doi: 10.3389/fncel.2013. 00156

Matricardi, S., Verrotti, A., Chiarelli, F., Cerminara, C., and Curatolo, P. (2014). Current advances in childhood absence epilepsy. Pediatr. Neurol. 50, 205-212. doi: 10.1016/j.pediatrneurol.2013.10.009

McCormick, D. A., and Contreras, D. (2001). On the cellular and network bases of epileptic seizures. Annu. Rev. Physiol. 63, 815-846. doi: 10.1146/annurev. physiol.63.1.815

Meeren, H., van Luijtelaar, G., Lopes da Silva, F., and Coenen, A. (2005). Evolving concepts on the pathophysiology of absence seizures: the cortical focus theory. Arch. Neurol. 62, 371-376. doi: 10.1001/archneur.62.3.371 
Meldrum, B. S., Akbar, M. T., and Chapman, A. G. (1999). Glutamate receptors and transporters in genetic and acquired models of epilepsy. Epilepsy Res. 36, 189-204. doi: 10.1016/S0920-1211(99)00051-0

Meng, H., Walker, N., Su, Y., and Qiao, X. (2006). Stargazin mutation impairs cerebellar synaptogenesis, synaptic maturation and synaptic protein distribution. Brain Res. 1124, 197-207. doi: 10.1016/j.brainres.2006.09.086

Menuz, K., and Nicoll, R. A. (2008). Loss of inhibitory neuron AMPA receptors contributes to ataxia and epilepsy in stargazer mice. J. Neurosci. 28, 10599-10603. doi: 10.1523/jneurosci.2732-08.2008

Paz, J., Bryant, A. S., Peng, K., Fenno, L., Yizhar, O., Frankel, W. N., et al. (2011). A new mode of corticothalamic transmission revealed in the Gria4-/- model of absence epilepsy. Nat. Neurosci. 14, 1167-1173. doi: 10.1038/nn.2896

Paz, J. T., and Huguenard, J. R. (2015). Microcircuits and their interactions in epilepsy: is the focus out of focus? Nat. Neurosci. 18, 351-359. doi: 10.1038/ nn.3950

Pelkey, K. A., Barksdale, E., Craig, M. T., Yuan, X., Sukumaran, M., Vargish, G. A., et al. (2015). Pentraxins coordinate excitatory synapse maturation and circuit integration of parvalbumin interneurons. Neuron 85, 1257-1272. doi: 10.1016/j.neuron.2015.02.020

Peters, A., Palay, S., and Webster, H. (1991). The Fine Structure of the Nervous System, 3rd Edn. Oxford: Oxford University Press.

Petersen, J. D., Chen, X., Vinade, L., Dosemeci, A., Lisman, J. E., and Reese, T. S. (2003). Distribution of postsynaptic density (PSD)-95 and Ca2+/calmodulindependent protein kinase II at the PSD. J. Neurosci. 23, 11270-11278.

Polack, P. O., Guillemain, I., Hu, E., Deransart, C., Depaulis, A., and Charpier, S. (2007). Deep layer somatosensory cortical neurons initiate spike-and-wave discharges in a genetic model of absence seizures. J. Neurosci. 27, 6590-6599. doi: 10.1523/jneurosci.0753-07.2007

Porter, J. T., Johnson, C. K., and Agmon, A. (2001). Diverse types of interneurons generate thalamus-evoked feedforward inhibition in the mouse barrel cortex. J. Neurosci. 21, 2699-2710.

Richardson, C. A., and Leitch, B. (2005). Phenotype of cerebellar glutamatergic neurons is altered in stargazer mutant mice lacking brain-derived neurotrophic factor mRNA expression. J. Comp. Neurol. 481, 145-159. doi: 10.1002/cne. 20386

Sasaki, S., Huda, K., Inoue, T., Miyata, M., and Imoto, K. (2006). Impaired feedforward inhibition of the thalamocortical projection in epileptic $\mathrm{Ca} 2+$ channel mutant mice, tottering. J. Neurosci. 26, 3056-3065. doi: 10.1523/ jneurosci.5422-05.2006

Sato, K., Kiyama, H., and Tohyama, M. (1993). The differential expression patterns of messenger RNAs encoding non-N-methyl-d-aspartate glutamate receptor subunits (GluR1-4) in the rat brain. Neuroscience 52, 515-539. doi: 10.1016/ 0306-4522(93)90403-3

Schubert, V., and Dotti, C. G. (2007). Transmitting on actin: synaptic control of dendritic architecture. J. Cell. Sci. 120, 205-212. doi: 10.1242/jcs.03337

Schwenk, J., Harmel, N., Zolles, G., Bildl, W., Kulik, A., Heimrich, B., et al. (2009). Functional proteomics identify cornichon proteins as auxiliary subunits of AMPA receptors. Science 323, 1313-1319. doi: 10.1126/science.1167852

Seeburg, P. H. (1993). The TINS/TiPS Lecture the molecular biology of mammalian glutamate receptor channels. Trends Neurosci. 16, 359-365.0. doi: 10.1016/ 0166-2236(93)90093-2
Seo, S., and Leitch, B. (2015). Synaptic changes in GABA receptor expression in the thalamus of the stargazer mouse model of absence epilepsy. Neuroscience 306, 28-38. doi: 10.1016/j.neuroscience.2015.08.021

Shevtsova, O., and Leitch, B. (2012). Selective loss of AMPA receptor subunits at inhibitory neuron synapses in the cerebellum of the ataxic stargazer mouse. Brain Res. 1427, 54-64. doi: 10.1016/j.brainres.2011.10.022

Snead, O. C. III (1995). Basic mechanisms of generalized absence seizures. Ann. Neurol. 37, 146-157. doi: 10.1002/ana.410370204

Tao, Y., Chen, Y. J., Shen, C., Luo, Z., Bates, C. R., Lee, D., et al. (2013). Erbin interacts with TARP ( -2 for surface expression of AMPA receptors in cortical interneurons. Nature Neurosci. 16, 290-299. doi: 10.1038/nn.3320

Timofeev, I., and Steriade, M. (2004). Neocortical seizures: initiation, development and cessation. Neuroscience 123, 299-336. doi: 10.1016/j.neuroscience.2003. 08.051

Tomita, S., Chen, L., Kawasaki, Y., Petralia, R. S., Wenthold, R. J., Nicoll, R. A., et al. (2003). Functional studies and distribution define a family of transmembrane AMPA receptor regulatory proteins. J. Cell Biol. 161, 805-881. doi: 10.1083/jcb. 200212116

Trinka, E., Steinhoff, B. J., Nikanorova, M., and Brodie, M. J. (2016). Perampanel for focal epilepsy: insights from early clinical experience. Acta Neurol. Scand. 133, 160-172. doi: 10.1111/ane.12529

Vandenberghe, W., Nicoll, R. A., and Bredt, D. S. (2005). Stargazin is an AMPA receptor auxiliary subunit. Proc. Natl. Acad. Sci. U.S.A. 102, 485-490. doi: 10.1073/pnas.0408269102

Wang, H. X., and Gao, W. J. (2010). Development of calcium-permeable AMPA receptors and their correlation with NMDA receptors in fast-spiking interneurons of rat prefrontal cortex. J. Physiol. 588, 2823-2838. doi: 10.1113/ jphysiol.2010.187591

Wirrel, E. C., Camfield, P. R., Camfield, C. S., Dooley, J. M., and Gordon, K. E. (1996). Accidental injury is a serious risk in children with typical absence epilepsy. Arch. Neurol. 53, 929-932. doi: 10.1001/archneur.1996. 00550090141020

Yamasaki, M., Fukaya, M., Yamazaki, M., Azechi, H., Natsume, R., Abe, M., et al. (2016). TARP gamma-2 and gamma-8 differentially control AMPAR density across schaffer collateral/commissural synapses in the hippocampal CA1 area. J. Neurosci. 36, 4296-4312. doi: 10.1523/jneurosci.4178-15.2016

Zimmerman, F. T., and Burgemeister, B. B. (1958). A new drug for petit mal epilepsy. Neurology 8, 769-775. doi: 10.1212/WNL.8.10.769

Conflict of Interest Statement: The authors declare that the research was conducted in the absence of any commercial or financial relationships that could be construed as a potential conflict of interest.

The reviewer HM and handling Editor declared their shared affiliation.

Copyright (c) 2017 Adotevi and Leitch. This is an open-access article distributed under the terms of the Creative Commons Attribution License (CC BY). The use, distribution or reproduction in other forums is permitted, provided the original author(s) or licensor are credited and that the original publication in this journal is cited, in accordance with accepted academic practice. No use, distribution or reproduction is permitted which does not comply with these terms. 